\title{
The first symptoms of cardiac reverse remodeling and clinical improvement after one-month, low-dose of Sacubitril-Valsartan therapy
}

\author{
Wioletta Sacharczuk ${ }^{1}$, Rafał Dankowski ${ }^{1}$, Anna Sowińska ${ }^{1}$, Artur Baszko ${ }^{1}$, and Andrzej \\ Szyszka ${ }^{1}$ \\ ${ }^{1}$ Poznan University of Medical Sciences
}

March 30, 2021

\begin{abstract}
Introduction: Little is known about reverse cardiac remodeling (CRR) under the low-dose S/V therapy. Methods: In 37 patients (mean age $64.5 \pm 17.5$ years, five females) $24 / 26 \mathrm{mg} \mathrm{S} / \mathrm{V}$ BID was started. After one month of observation, the following CRR parameters improved: LVEDD (-[?]2,9+-2.6mm, p<0.01), LVESD (-[?] 2,4+-2.5mm, p<0.01), LVEDV(-[?]14.6+-33.1ml, $\mathrm{p}=0.04)$, LVESV (-[?]13.4+-30.6ml, $\mathrm{p}=0.04)$, LAVI $(-[?] 8.7+-37.7 \mathrm{ml} / \mathrm{m} 2, \mathrm{p}<0.01)$, and EROA $(-[?] 0.09+-0,01 \mathrm{~cm} 2 ; \mathrm{p}=0.03)$. In opposite to LVEF global longitudinal strain (GLS) changed from $-6.6 \%$ to $-7.9 \%$ (absolute improvement of $16 \%, \mathrm{p}<0.001$ ). Walked distance in 6-MWT $(+[?] 65.4+-75.8 \mathrm{~m}, \mathrm{p}<0.001)$, and the quality of life (MLHFQ 22 vs 16 scores, $\mathrm{p}<0.01)$ improved. Decreasing NT-proBNP (-[?] 1,203,1+-3,121,4pg/ml, p=0.03) and troponin T (-[?] 4.7+-9.4pg/ml, p=0.004) were observed. Correlation between GLS and LVESV $(r=-0,43, p=0.027)$ was found. ROC curve analysis showed that GLS cut-off value $-8 \%$ is a good predictor of clinical improvement (6MWT: AUC $0.69 \mathrm{p}=0.04$ ) and CRR (MRvol: AUC:0.74 p=0.01; LAVI: AUC 0.71 $\mathrm{p}=0.04)$. Conclusion: One-month, low-dose (24/26 mg BID) S/V therapy initiates CRR. GLS's ability to evaluate LV function is better than LVEF's. S/V should be started early as patients with symptomatic HFrEF and less impaired LV systolic function (GLS $<-8 \%)$ are more likely to develop CRR and clinical improvement.
\end{abstract}

\section{Hosted file}

SV BODY TEXT_JCII.pdf available at https://authorea.com/users/391008/articles/516029-thefirst-symptoms-of-cardiac-reverse-remodeling-and-clinical-improvement-after-one-monthlow-dose-of-sacubitril-valsartan-therapy 

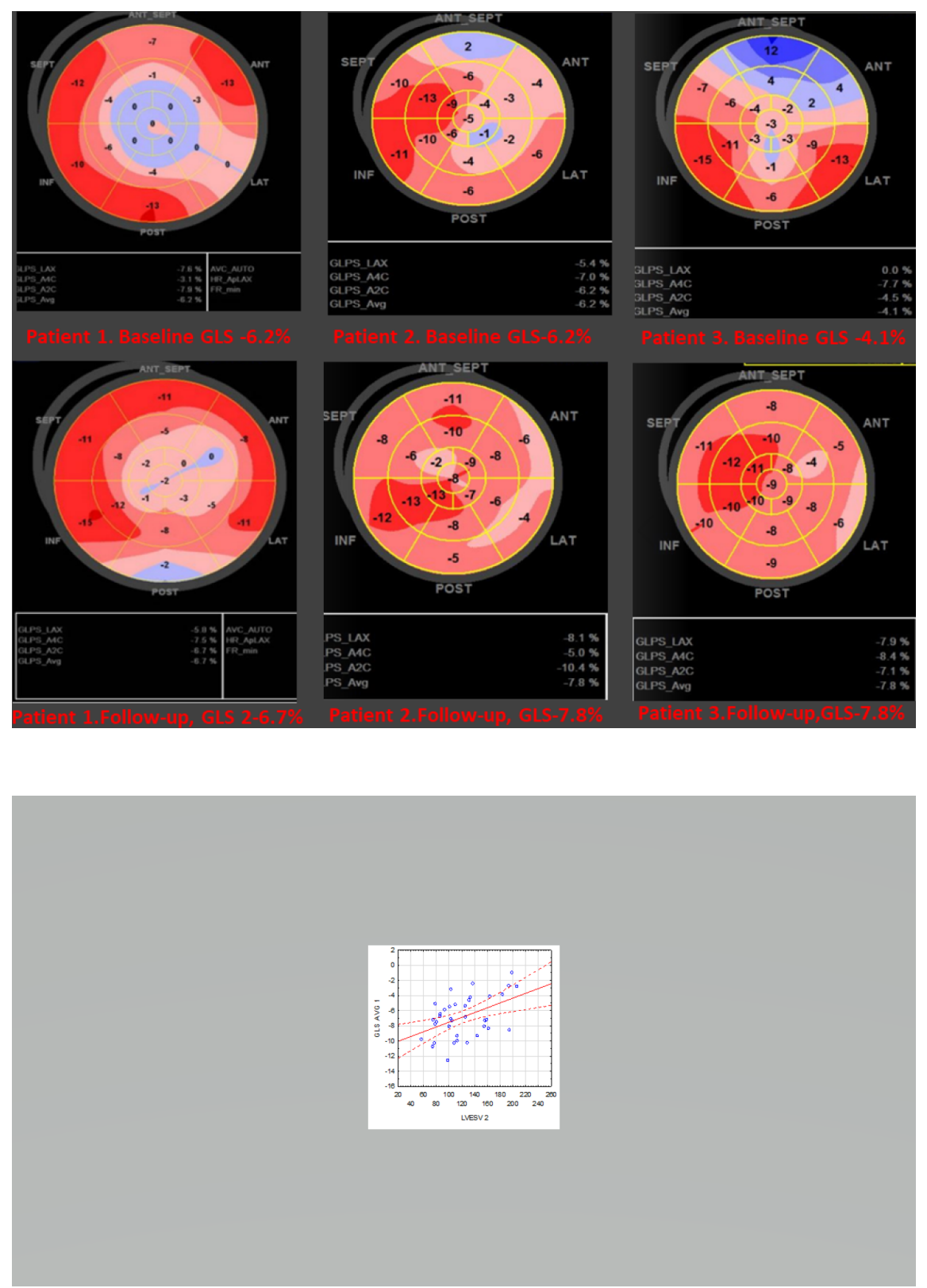


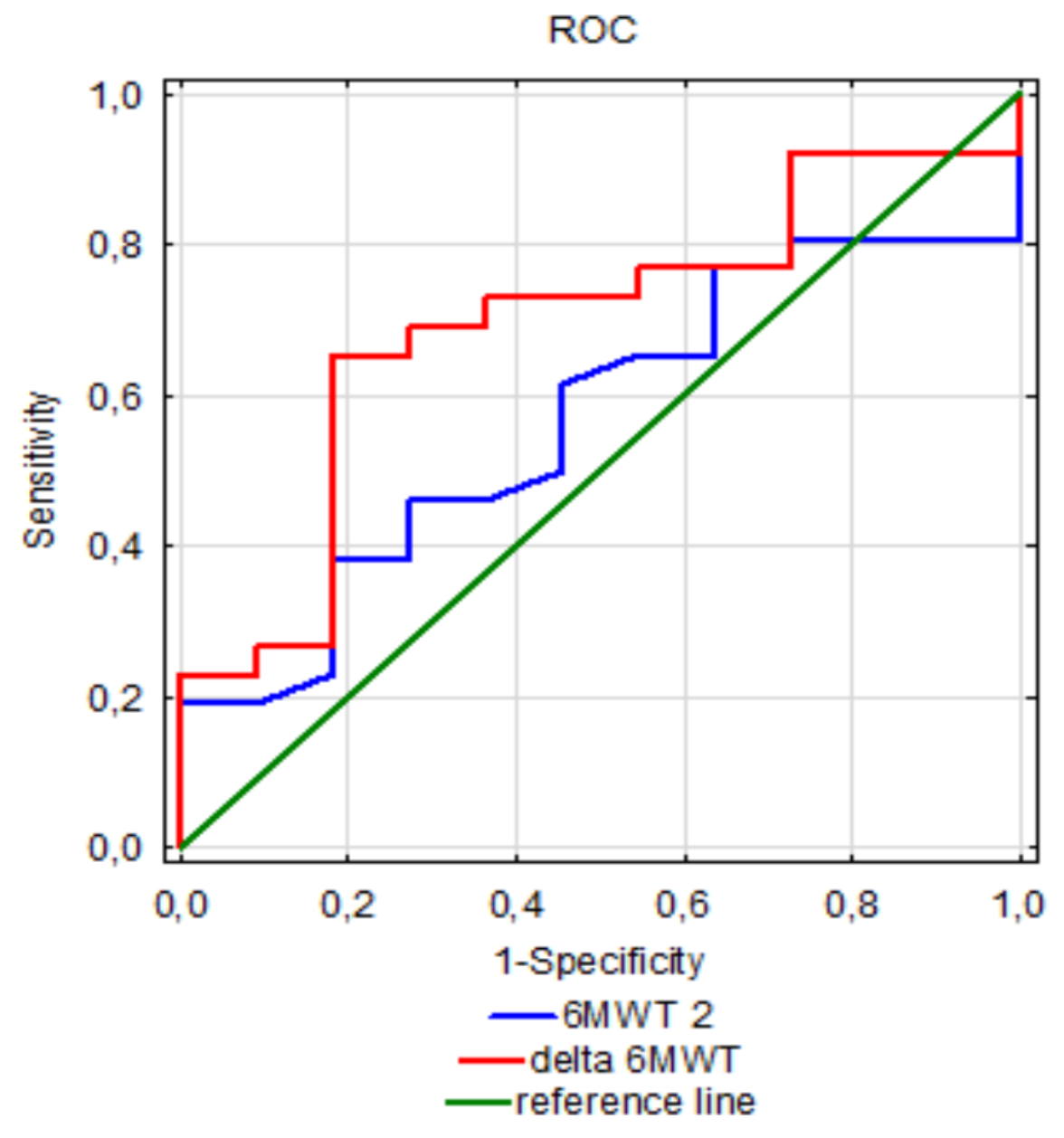




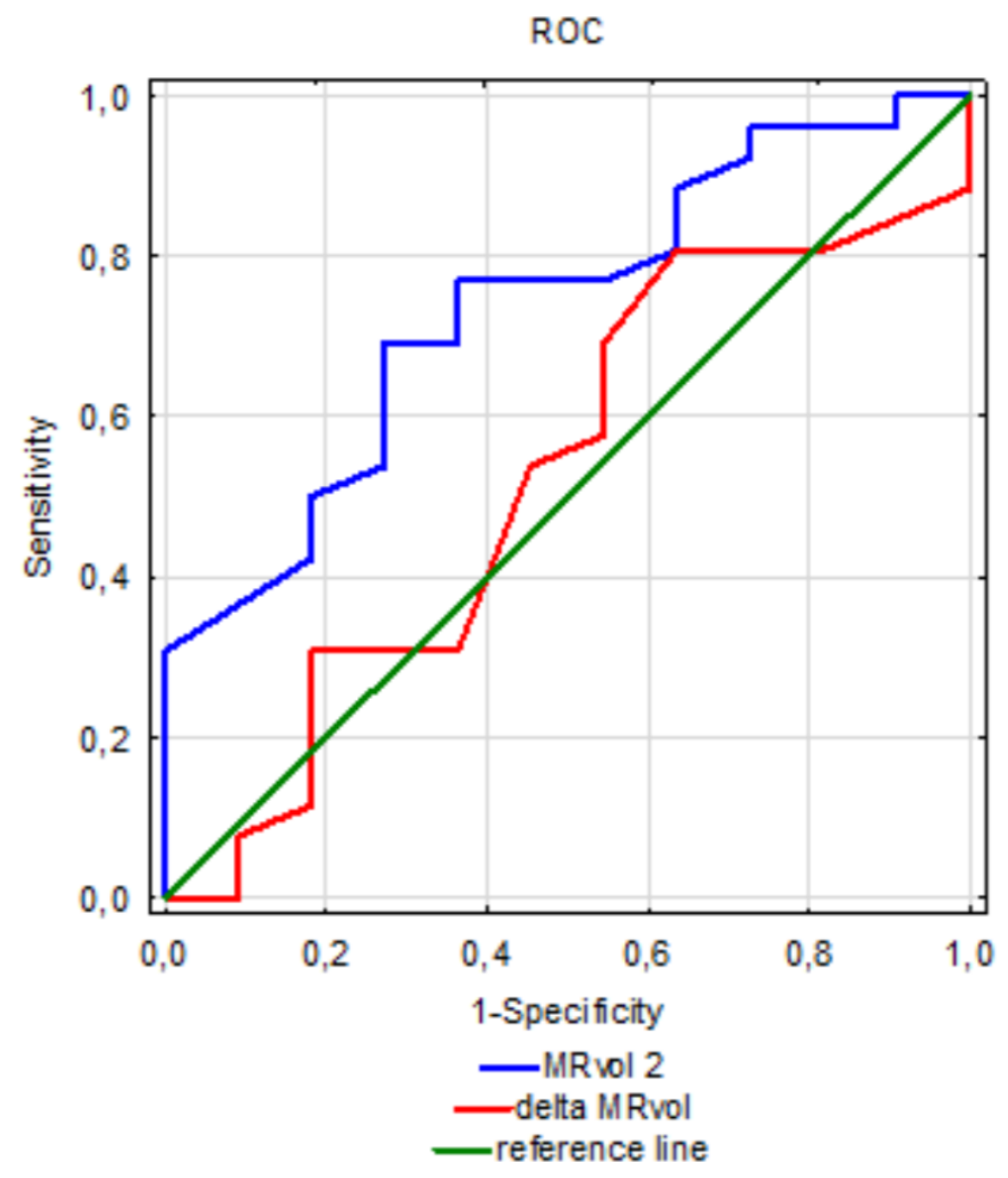




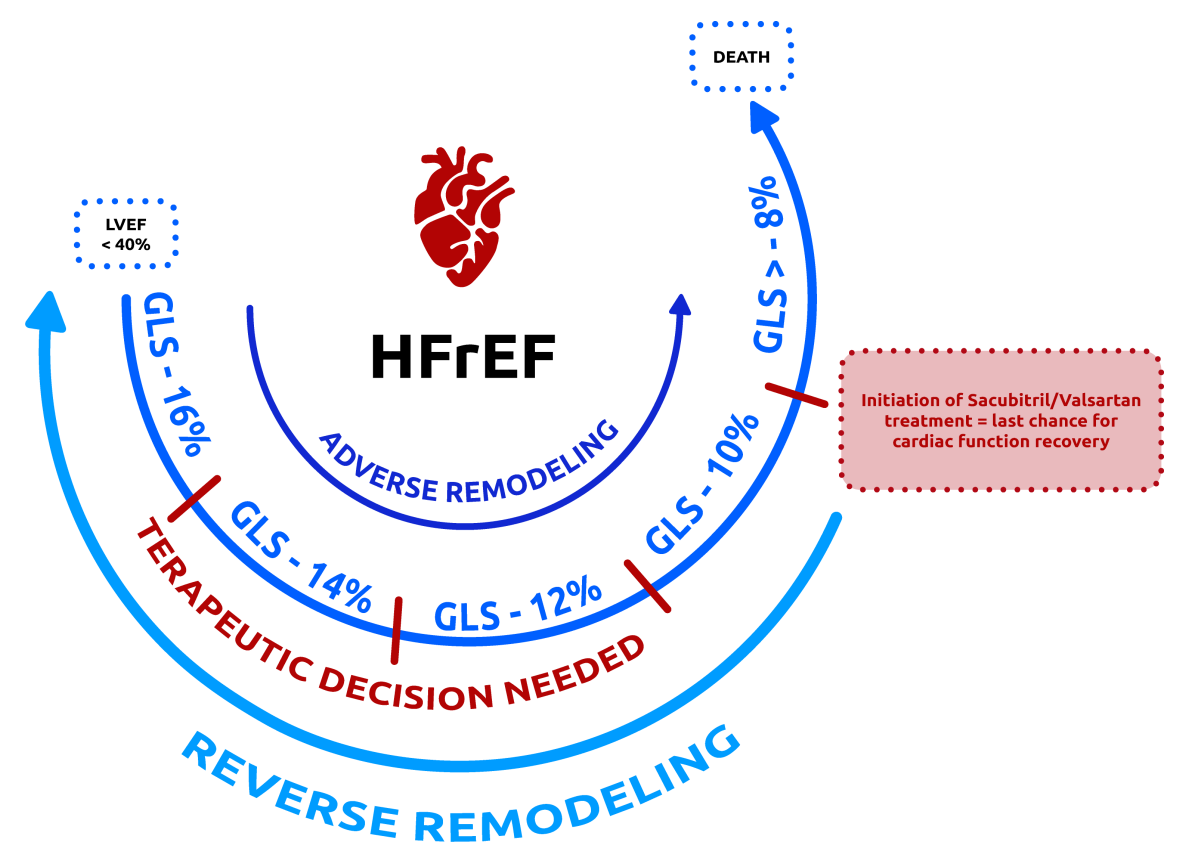

